\title{
Two new families of non-CCA groups*
}

\author{
Brandon Fuller (D), Joy Morris ${ }^{\dagger}$ \\ Department of Mathematics and Computer Science, University of Lethbridge \\ Lethbridge, AB T1K 3M4, Canada
}

Received 24 May 2020, accepted 11 August 2020, published online 28 January 2021

\begin{abstract}
We determine two new infinite families of Cayley graphs that admit colour-preserving automorphisms that do not come from the group action. By definition, this means that these Cayley graphs fail to have the CCA (Cayley Colour Automorphism) property, and the corresponding infinite families of groups also fail to have the CCA property. The families of groups consist of the direct product of any dihedral group of order $2 n$ where $n \geq 3$ is odd, with either itself, or the cyclic group of order $n$. In particular, this family of examples includes the smallest non-CCA group that does not fit into any previous family of known non-CCA groups.
\end{abstract}

Keywords: Cayley graphs, automorphisms, colour preserving, CCA

Math. Subj. Class.: 05C25

\section{Introduction}

All groups and graphs in this paper are finite. All of our graphs are simple, undirected, and have no loops.

A Cayley graph of $G$ with respect to $C$ (a subset of $G \backslash\{e\}$ ) is the graph $\operatorname{Cay}(G, C)$ whose vertices are the elements of $G$, with an edge from $g$ to $g c$ if and only if $g \in G, c \in$ $C$. The set $C$ is known as the connection set of $\operatorname{Cay}(G, C)$. This connection set gives a natural colouring of the edges where we colour the edge from $g$ to $g c$ (which is the same as the edge from $g c$ to $g$ ) with a colour associated to $\left\{c, c^{-1}\right\}$. A colour-preserving automorphism of $\operatorname{Cay}(G, C)$ is a permutation of the vertices that preserves edges and nonedges as well as edge colour. A Cayley graph $\operatorname{Cay}(G, C)$ is said to have the Cayley Colour

\footnotetext{
* The authors thank the anonymous referees for their careful reading of this paper, and for their helpful suggestions.

${ }^{\dagger}$ This work was supported by the Natural Science and Engineering Research Council of Canada (grant RGPIN2017-04905).

E-mail addresses: brandon.fuller621@gmail.com (Brandon Fuller), joy.morris@uleth.ca (Joy Morris)
} 
Automorphism (CCA) property if every colour-preserving automorphism of the graph is an affine function on $G$. The group $G$ is said to be CCA if every connected Cayley graph of $G$ is CCA.

The study of this property has only come up recently in history. In 2012, M. Conder, T. Pizanski and A. Žitnik [1] proposed a question about the permutations of circulant graphs that preserve a certain edge colouring that the second author [7] answered. The second author showed that for any connected Cayley graph on the cyclic group $C_{n}$, all colour-preserving automorphisms that fix the identity are automorphisms of $C_{n}$. In 2014, A. Hujdurović, K. Kutnar, D. W. Morris, and J. Morris [3] extended the original question by looking at Cayley graphs, using the natural edge colouring described. In early 2017, L. Morgan, J. Morris and G. Verret [5, 6] gave new results for finite simple groups and Sylow cyclic groups that generalized results produced by E. Dobson, A. Hujdurović, K. Kutnar, and J. Morris in [2]. The problem of determining colour-preserving and colourpermuting automorphisms for directed Cayley graphs has already been studied and is well understood: see for example [10], where the authors showed that for a connected Cayley digraph, every colour-preserving automorphism is a left-translation by some element of the group.

In his M.Sc. thesis, the first author produced code using GAP [9] and Sage [8] that determines whether or not a group or graph has the CCA property, and ran this code on all groups of order up to 200 (excluding orders 128 and 192). With this data in hand, a logical step was to look for theoretical methods to explain some of the small non-CCA groups that were not previously understood, and if possible to find new infinite families of non-CCA groups using this method.

In this paper, we use results from [5] to show that whenever $n \geq 3$, the groups $C_{n} \times D_{2 n}$ and $D_{2 n} \times D_{2 n}$ are non-CCA groups. Section 2 contains some basic background, definitions, and notation, along with the statements of the results we need from [5]. Section 3 provides proofs of our main results.

\section{Background}

The following notation is used for the remainder of this paper. We use $C_{n}$ to represent the cyclic group of order $n$, and $D_{2 n}$ (for $n \geq 3$ ) to represent the dihedral group of order $2 n$. We also have $Q_{8}$ as the quaternion group of order 8 .

The notation $\Gamma=(V(\Gamma), E(\Gamma))$ represents a graph of finite order, consisting of a set $V=V(\Gamma)$ of vertices and a set $E=E(\Gamma) \subseteq\{\{u, v\} \mid u, v \in V\}$ of edges. The set of vertices that are adjacent to a vertex $v$, denoted $\Gamma(v)$, is called the neighbourhood of $v$. We use $\mathcal{L}(\Gamma)$ to indicate the line graph of the graph $\Gamma$, and $\mathcal{S}(\Gamma)$ is the subdivision graph of the graph $\Gamma$.

If $G$ acts on a graph $\Gamma$ and $S \subseteq V(\Gamma)$ is fixed setwise under the action of $G$, then $G^{S}$ is the restriction of the action of $G$ to $S$. We use $G_{v}$ to denote the stabiliser subgroup (elements of $G$ that fix $v$ ).

Definition 2.1 ([3, Definition. 2.6]). For an abelian group $A$ of even order and an involution $y \in A$, the corresponding generalized dicyclic group is

$$
\operatorname{Dic}(A, y)=\left\langle x, A \mid x^{2}=y, x^{-1} a x=a^{-1}, \forall a \in A\right\rangle .
$$


Definition 2.2 ([3, Definition. 5.1]). The generalized dihedral group over an abelian group $A$ is the group

$$
\operatorname{Dih}(A)=\left\langle\sigma, A \mid \sigma^{2}=e, \sigma a \sigma=a^{-1}, \forall a \in A\right\rangle
$$

Definition 2.3 ([5, Definition 4.5]). Let $B$ be a permutation group and $G$ a regular subgroup of $B$. Let $\mathcal{A}^{0}$ be the colour-preserving automorphism group of the complete Cayley colour graph $K_{G}=\operatorname{Cay}(G, G \backslash\{e\})$, and let $\widehat{G}$ be the subgroup of $\mathcal{A}^{0}$ consisting of all left translations by elements of $G$. We say that $(G, B)$ is a complete colour pair if $B$ is a subgroup of $\mathcal{A}^{0}$ and $G$ is one of the following:

- $G$ is abelian but not an elementary abelian 2-group, and $\mathcal{A}^{0} \cong \operatorname{Dih}(G)$.

- $G \cong \operatorname{Dic}(A, y)$ but not of the form $Q_{8} \times C_{2}^{n}$ and $\mathcal{A}^{0}=\widehat{G} \rtimes\langle\sigma\rangle$, where $\sigma$ is the permutation that fixes $A$ pointwise and maps every element of the coset $A x$ to its inverse.

- $G \cong Q_{8} \times C_{2}^{n}$ and $\mathcal{A}^{0}=\left\langle\widehat{G}, \sigma_{i}, \sigma_{j}, \sigma_{k}\right\rangle$, where $\sigma_{\alpha}$ is the permutation of $Q_{8} \times C_{2}^{n}$ that inverts every element of $\{ \pm \alpha\} \times C_{2}^{n}$ and fixes every other element.

The importance of Definition 2.3 comes from the fact that if $(G, B)$ is a complete colour pair, then in each case we have a colour-preserving automorphism of $K_{G}$ that is not an element of $\widehat{G}$.

An arc is an orientation for an edge in a graph. So the edge $\{u, v\}$ admits two possible orientations: $(u, v)$, or $(v, u)$.

Definition 2.4. Let $\Gamma$ be a graph and $G$ a permutation group acting on the vertices of $\Gamma$. We say that $\Gamma$ is a $\boldsymbol{G}$-arc-regular graph if for each pair of $\operatorname{arcs} e_{1}=(u, v)$ and $e_{2}=(w, x)$ (each an oriented edge from $E(\Gamma)$ ), there exists a unique element of $G$ that maps $u$ to $w$ and $v$ to $x$, so that it maps the chosen orientation for $e_{1}$ to the chosen orientation for $e_{2}$.

Notation 2.5. For the remainder of this paper we use the following notation. Consider the complete bipartite graph $K_{n, n}$. We define $\rho_{1}$ to be a cyclic permutation on one of the bipartition sets, and $\rho_{2}$ be a cyclic permutation on the other bipartition set, with $\tau$ an involution that commutes with $\rho_{1} \rho_{2}$ and switches the bipartition sets. Let $\sigma_{1}$ be an involution acting on the first bipartition set that inverts $\rho_{1}$, and $\sigma_{2}$ an involution acting on the second bipartition set that inverts $\rho_{2}$.

We label the edges of $\mathcal{S}\left(K_{n, n}\right)$ as follows. Use $v$ to denote the unique vertex in the second bipartition set of $K_{n, n}$ that is fixed under the action of $\sigma_{2}$. Now in $\mathcal{S}\left(K_{n, n}\right)$ label the edge from $\tau(v)$ to the vertex subdividing $\{v, \tau(v)\}$ with the identity element $e$ of $G$, and label each other edge by the unique element of $G$ that maps the edge $e$ to that edge. This produces a labeling that shows us that $\mathcal{L}\left(\mathcal{S}\left(K_{n, n}\right)\right)$ is a Cayley graph on $G$. From this it is straightforward to observe that the connection set $C$ (which consists of all neighbours of $e)$ is $\{\tau\} \cup\left\{\rho_{2}^{i}: 1 \leq i \leq n-1\right\}$.

Corollary 2.6 ([5, Corollary 4.10]). Let $\Gamma$ be a connected $G$-arc-regular graph. If $H$ is a group of automorphisms of $\Gamma$ such that:

- $G \leq H$, and

- $\left(G_{v}^{\Gamma(v)}, H_{v}^{\Gamma(v)}\right)$ is a complete colour pair for every vertex $v$ of $\Gamma$, 
then $H$ is a colour-preserving group of automorphisms of $\mathcal{L}(\mathcal{S}(\Gamma))$ viewed as a Cayley graph on $G$.

The real point of this corollary is that if we show that some element of $H$ is not an affine function, then this implies that $\mathcal{L}(\mathcal{S}(\Gamma))$ is a non-CCA graph, and so $G$ is a nonCCA group. The fact that $\left(G_{v}^{\Gamma(v)}, H_{v}^{\Gamma(v)}\right)$ is a complete colour pair is what allows us to produce the desired non-affine element of $H$.

\section{Main results}

In our main result, we show that $K_{n, n}$ is a (connected) $C_{n} \times D_{2 n}$-arc-regular graph and therefore if we take $\Gamma=K_{n, n}, G=C_{n} \times D_{2 n}$, and $H=D_{2 n} \curlywedge C_{2}$ then all of the conditions of Corollary 2.6 are satisfied. For clarity, we are using $D_{2 n} \prec C_{2}$ to denote the semidirect product $\left(D_{2 n} \times D_{2 n}\right) \rtimes C_{2}$, where the $C_{2}$ is acting on the coordinates in the direct product. Hence $D_{2 n}<C_{2}$ is a colour-preserving group of automorphisms of $\mathcal{L}\left(\mathcal{S}\left(K_{n, n}\right)\right)$. With this we find an element in $D_{2 n}$ \& $C_{2}$, a colour-preserving automorphism, that is a non-affine function to show that $\mathcal{L}\left(\mathcal{S}\left(K_{n, n}\right)\right)$ is non-CCA. The proof is not particularly difficult; the difficulty of this result lies in finding an arc-regular graph and corresponding permutation groups to which we can apply Corollary 2.6.

Theorem 3.1. The graph $\mathcal{L}\left(\mathcal{S}\left(K_{n, n}\right)\right)$ viewed as a Cayley graph on $C_{n} \times D_{2 n}$ is non-CCA whenever $n \geq 3$ is odd.

Specifically, if $G=\left\langle\rho_{1}, \rho_{2}, \tau\right\rangle$ and $C=\{\tau\} \cup\left\{\rho_{2}^{i}: 1 \leq i \leq n-1\right\}$, then $\sigma_{2}$ is a non-affine colour-preserving automorphism of $\mathrm{Cay}(G, C)$.

Proof. We use Notation 2.5 and the labelling that is given in the paragraph following that notation to view $\mathcal{L}(\mathcal{S}(\Gamma))$ as a Cayley graph on $G$. Observe that $G=\left\langle\rho_{1}, \rho_{2}, \tau\right\rangle=$ $\left\langle\rho_{1} \rho_{2}, \rho_{1} \rho_{2}^{-1}, \tau\right\rangle \cong C_{n} \times D_{2 n}$ since $n$ is odd so that $\left\langle\rho_{2}^{2}\right\rangle=\left\langle\rho_{2}\right\rangle$. Notice that $G$ acts regularly on the arcs of $K_{n, n}$, so that $K_{n, n}$ is $G$-arc-regular.

Consider now the group $H=\left\langle\rho_{1}, \rho_{2}, \tau, \sigma_{1}, \sigma_{2}\right\rangle \cong D_{2 n}$ 乙 $C_{2}$ where each copy of $D_{2 n}$ acts independently on one of the bipartition sets of $K_{n, n}$, and the $C_{2}$ (generated by $\tau$ ) exchanges the coordinates. The first copy of $D_{2 n}$ is generated by $\rho_{1}$ and $\sigma_{1}$. The second copy is generated by $\rho_{2}$ and $\sigma_{2}$. It is clear that $G \leq H$ since $\rho_{1}, \rho_{2}, \tau \in H$.

Let $v$ be an arbitrary vertex of the second bipartition set. The neighbours of $v$ are all the elements of the first bipartition set. We notice that $G_{v}^{\Gamma(v)}$ is the subgroup of $G$ that fixes $v$ and its action is restricted to the bipartition set that $v$ is not in. We see that $\rho_{1}$ is the cyclic permutation of $\Gamma(v)$. Since $G=\left\langle\rho_{1}, \rho_{2}, \tau\right\rangle$, it is not hard to observe that $G_{v}^{\Gamma(v)}=\left\langle\rho_{1}\right\rangle \cong$ $C_{n}$. Similarily since $H=\left\langle\rho_{1}, \rho_{2}, \tau, \sigma_{1}, \sigma_{2}\right\rangle$ we have that $H_{v}^{\Gamma(v)}=\left\langle\rho_{1}, \sigma_{1}\right\rangle \cong D_{2 n}$. Thus we only need to show that $\left(C_{n}, D_{2 n}\right)$ is a complete colour pair.

We can see $\left(C_{n}, D_{2 n}\right)$ is a complete colour pair using Definition 2.3. Let $\mathcal{A}^{0}$ be the colour-preserving automorphism group for the Cayley graph $K_{G_{v}^{\Gamma(v)}}$. We know that $\mathcal{A}^{0}=$ $D_{2 n}=\operatorname{Dih}\left(C_{n}\right)$ and thus since $C_{n}$ is abelian and is not an elementary abelian 2-group $(n \geq 3$ ), all the properties of the first possibility for a complete colour pair are met. (In this case, $B=D_{2 n}=\mathcal{A}^{0}$.) We thus conclude (using Corollary 2.6) that every element of $H$ is a colour-preserving automorphism of $\mathcal{L}\left(\mathcal{S}\left(K_{n, n}\right)\right)$ viewed as a Cayley graph on $G$.

It remains to show that some element of $H$ is not affine. We claim that $\sigma_{2}$ (acting on $G$ as an automorphism of the Cayley graph) is such an element. In order to prove this, we show that $\sigma_{2}^{-1} \tau \sigma_{2}$ is not an element of $G$. Let $v$ be the unique vertex in the second 
bipartition set that is fixed by $\sigma_{2}$. Clearly, $\sigma_{2}^{-1} \tau \sigma_{2}=\sigma_{2} \tau \sigma_{2}$ maps the arc $(v, \tau(v))$ to the $\operatorname{arc}(\tau(v), v)$, since $\sigma_{2}$ fixes both $v$ and $\tau(v)$. Since $G$ is acting arc-regularly, it has a unique element that maps $(v, \tau(v))$ to the arc $(\tau(v), v)$, and we know that this element is $\tau$. So if $\sigma_{2}$ normalises $G$, we must have $\sigma_{2} \tau \sigma_{2}=\tau$. It is straightforward to verify that this is not the case. For example, $\tau \rho_{2}(v)=\tau \rho_{1} \rho_{2}(v)=\rho_{1} \rho_{2} \tau(v)=\rho_{1} \tau(v)$ (the first equality follows from the fact that $\rho_{1}$ fixes the bipartition set that contains $v$; the second equality from the fact that $\tau$ and $\rho_{1} \rho_{2}$ commute, and the third from the fact that $\rho_{2}$ fixes the bipartition set that does not contain $v$ ). However, $\sigma_{2} \tau \sigma_{2} \rho_{2}(v)=\tau \sigma_{2} \rho_{2}(v)=\tau \rho_{2}^{-1} \sigma_{2}(v)=\tau \rho_{2}^{-1}(v)$ (the first equality follows because $\sigma_{2}$ fixes the bipartition set that does not contain $v$; the second because $\left\langle\sigma_{2}, \rho_{2}\right\rangle \cong D_{2 n}$, so $\sigma_{2}$ inverts $\rho_{2}$; and the third because $\sigma_{2}$ fixes $v$ ). However, since $n \geq 3, \tau \rho_{2}^{-1}(v)$ is not the same as $\tau \rho_{2}(v)$, because the order of $\rho_{2}$ is $n$. Thus, $\sigma_{2} \in H$ does not normalise $G$, as claimed.

Corollary 3.2. The group $C_{n} \times D_{2 n}$ is non-CCA whenever $n \geq 3$ is odd.

We use the above result to show that $D_{2 n} \times D_{2 n}$ is not CCA whenever $n \geq 3$ is odd.

Proposition 3.3. The group $D_{2 n} \times D_{2 n}$ is non-CCA whenever $n \geq 3$ is odd.

Proof. Let $G=\left\langle\rho_{1}, \rho_{2}, \tau\right\rangle$ where these permutations are as defined in Notation 2.5. Define $H=\langle G, \gamma\rangle$, where $\gamma$ is an involution that commutes with $\tau$ and with $\rho_{1}^{-1} \rho_{2}$, and inverts $\rho_{1} \rho_{2}$. Notice that this implies $H \cong D_{2 n} \times D_{2 n}$.

By Theorem 3.1, if $G=\left\langle\rho_{1}, \rho_{2}, \tau\right\rangle$ and $C=\{\tau\} \cup\left\{\rho_{2}^{i}: 1 \leq i \leq n-1\right\}$, then $\sigma_{2}$ is a non-affine automorphism of $\operatorname{Cay}(G, C$ ) (in its action on $G$ as an automorphism of this Cayley graph). We use this to produce a non-affine colour-preserving automorphism $\varphi$ on $\Gamma=\operatorname{Cay}(H, C \cup\{\gamma\})$.

Define $\varphi$ by $\varphi(g)=\sigma_{2}(g)$, and $\varphi(g \gamma)=\sigma_{2}(g) \gamma$ for every $g \in G$. We first show that $\varphi$ is colour-preserving on $\Gamma$.

Consider any edge $e$ of $\Gamma$. If both endpoints of $e$ are in $G$ then $\varphi(e)=\sigma_{2}(e)$ and since $\sigma_{2}$ preserves colours, so does $\varphi$.

If one endpoint of $e$ is in $G$ and the other is not, then it must be the case that $e$ is coloured $\gamma$, and its endpoints are $g$ and $g \gamma$ for some $g \in G$. Furthermore, by definition of $\varphi$ we have $\varphi(g \gamma)=\varphi(g) \gamma$, so there is an edge between $\varphi(g)$ and $\varphi(g \gamma)$, and its colour is $\gamma$. Thus $\varphi$ also preserves the colour of any such edge.

The final case to consider is if both endpoints of $e$ are in $G \gamma$. Suppose the endpoints of $e$ are $\rho_{1}^{i_{1}} \rho_{2}^{i_{2}} \tau^{f_{1}} \gamma$ and $\rho_{1}^{j_{1}} \rho_{2}^{j_{2}} \tau^{f_{2}} \gamma$, where $0 \leq i_{1}, i_{2}, j_{1}, j_{2} \leq n-1$, and $0 \leq f_{1}, f_{2} \leq 1$. Since there is an edge between these vertices, we must have $\gamma \tau^{f_{1}} \rho_{1}^{j_{1}-i_{i}} \rho_{2}^{j_{2}-i_{2}} \tau^{f_{2}} \gamma \in C$ (recall that $\gamma$ and $\tau$ are both involutions). Note that $\rho_{1}^{a} \rho_{2}^{b}=\left(\rho_{1} \rho_{2}\right)^{(a+b) / 2}\left(\rho_{1}^{-1} \rho_{2}\right)^{(b-a) / 2}$; we want to use this because we know that $\gamma$ commutes with $\tau$ and with $\rho_{1}^{-1} \rho_{2}$ but inverts $\rho_{1} \rho_{2}$. So we have

$$
\begin{gathered}
\gamma \tau^{f_{1}}\left(\rho_{1} \rho_{2}\right)^{\left(j_{1}+j_{2}-i_{1}-i_{2}\right) / 2}\left(\rho_{1}^{-1} \rho_{2}\right)^{\left(j_{2}+i_{1}-i_{2}-j_{1}\right) / 2} \tau^{f_{2}} \gamma \\
=\tau^{f_{1}}\left(\rho_{1} \rho_{2}\right)^{\left(i_{1}+i_{2}-j_{1}-j_{2}\right) / 2}\left(\rho_{1}^{-1} \rho_{2}\right)^{\left(j_{2}+i_{1}-i_{2}-j_{1}\right) / 2} \tau^{f_{2}} \\
=\tau^{f_{1}} \rho_{1}^{i_{2}-j_{2}} \rho_{2}^{i_{1}-j_{1}} \tau^{f_{2}} \in C .
\end{gathered}
$$

Since we know the elements of $C$, this implies one of three possibilities:

- the element is $\tau$, so that $i_{2}=j_{2}$ and $i_{1}=j_{1}$, and $\left\{f_{1}, f_{2}\right\}=\{0,1\}$; 
- $f_{1}=f_{2}=0$ and the element is $\rho_{2}^{j}$ for some $1 \leq j \leq n-1$, so $i_{2}=j_{2}$, and $\left.j=i_{1}-j_{1}\right)$; or

- $f_{1}=f_{2}=1$ and the element is $\rho_{2}^{j}$ for some $1 \leq j \leq n-1$, so (using the above equation and the fact that $\tau$ commutes with $\rho_{1} \rho_{2}$ and inverts $\left.\rho_{1}^{-1} \rho_{2}\right) i_{1}=j_{1}$, and $j=j_{2}-i_{2}$.

We now need to understand the images of the endpoints of $e$ under $\varphi$. Recall from the labelling established immediately following Notation 2.5 that we choose the vertex $v$ to be the unique vertex in the second bipartition set that is fixed by $\sigma_{2}$, and in $\mathcal{S}\left(K_{n, n}\right)$ we label the edge from $\tau(v)$ to the vertex subdividing $\{v, \tau(v)\}$ with the identity element of $G$. This means that the edge from $\rho_{1}^{i_{1}} \tau(v)$ to the vertex subdividing $\left\{\rho_{1}^{i_{1}} \tau(v), \rho_{2}^{i_{2}}(v)\right\}$ will be the image of the edge labelled with the identity under the action of $\rho_{1}^{i_{1}} \rho_{2}^{i_{2}}$, so is labelled $\rho_{1}^{i_{1}} \rho_{2}^{i_{2}}$. Similarly, since the edge from $v$ to the vertex subdividing $\{v, \tau(v)\}$ has the label $\tau$, the edge from $\rho_{2}^{i_{2}}(v)$ to the vertex subdividing $\left\{\rho_{1}^{i_{1}} \tau(v), \rho_{2}^{i_{2}}(v)\right\}$ will be labelled $\rho_{1}^{i_{1}} \rho_{2}^{i_{2}} \tau$. This is the other "half" of the same subdivided edge from $K_{n, n}$. It should now be apparent that $\sigma_{2}\left(\rho_{1}^{i_{1}} \rho_{2}^{i_{2}}\right)=\rho_{1}^{i_{1}} \rho_{2}^{-i_{2}}$ and therefore $\sigma_{2}\left(\rho_{1}^{i_{1}} \rho_{2}^{i_{2}} \tau\right)=\rho_{1}^{i_{1}} \rho_{2}^{-i_{2}} \tau$ (the other half of the same subdivided edge from $K_{n, n}$ ). Thus, the images of the endpoints of $e$ under $\varphi$ are $\rho_{1}^{i_{1}} \rho_{2}^{-i_{2}} \tau^{f_{1}} \gamma$ and $\rho_{1}^{j_{1}} \rho_{2}^{-j_{2}} \tau^{f_{2}} \gamma$.

Now using similar calculations to those above, the colour of the edge between these images is

$$
\tau^{f_{1}} \rho_{1}^{j_{2}-i_{2}} \rho_{2}^{i_{1}-j_{1}} \tau^{f_{2}}
$$

(together with its inverse). Taking the three possibilities identified above in turn, if $i_{1}=j_{1}$, $i_{2}=j_{2}$, and $\left\{f_{1}, f_{2}\right\}=\{0,1\}$ then this colour is $\tau$ as before, so $\varphi$ has preserved the colour. If $f_{1}=f_{2}=0, i_{2}=j_{2}$, and the colour of $e$ was $\left\{\rho_{2}^{j}, \rho_{2}^{-j}\right\}$ where $j=i_{1}-j_{1}$, then the colour of this edge is also $\left\{\rho_{2}^{j}, \rho_{2}^{-j}\right\}$. Finally, if $f_{1}=f_{2}=1, i_{1}=j_{1}$, and the colour of $e$ was $\left\{\rho_{2}^{j}, \rho_{2}^{-j}\right\}$ where $j=j_{2}-i_{2}$, then the colour of this edge is $\left\{\rho_{2}^{j}, \rho_{2}^{-j}\right\}$. So in all cases the colour of $e$ is preserved under the action of $\varphi$. This completes the proof that $\varphi$ is colour-preserving.

Since $\operatorname{Cay}(H, C)$ has two connected components (on $G$ and $G \gamma$ ), any colour-preserving automorphism of $\Gamma$ must preserve these components. Therefore, if $\varphi$ is affine then its restriction to $G$ (which is $\sigma_{2}$ ) would have to be affine on $G$. By Theorem 3.1 this is not the case. Thus, $\varphi$ is a colour-preserving automorphism of $\Gamma$ that is not affine, and therefore $\Gamma$ and $H$ are not CCA.

\section{ORCID iDs}

Brandon Fuller (D) https://orcid.org/0000-0002-0757-8097

Joy Morris (D) https://orcid.org/0000-0003-2416-669X

\section{References}

[1] M. D. E. Conder, T. Pisanski and A. Žitnik, GI-graphs: a new class of graphs with many symmetries, J. Algebraic Combin. 40 (2014), 209-231, doi:10.1007/s10801-013-0484-3.

[2] E. Dobson, A. Hujdurović, K. Kutnar and J. Morris, On color-preserving automorphisms of Cayley graphs of odd square-free order, J. Algebraic Combin. 45 (2017), 407-422, doi:10. 1007/s10801-016-0711-9.

[3] A. Hujdurović, K. Kutnar, D. W. Morris and J. Morris, On colour-preserving automorphisms of Cayley graphs, Ars Math. Contemp. 11 (2016), 189-213, doi:10.26493/1855-3974.771.9b3. 
[4] J. Koolen, J. Kwak and M. Xu, Applications of Group Theory to Combinatorics, CRC Press, 2008, https: / /books.google.com/books?id=4ayMmAEACAAJ.

[5] L. Morgan, J. Morris and G. Verret, Characterising CCA Sylow cyclic groups whose order is not divisible by four, Ars. Math. Contemp. 14 (2018), 83-95, doi:10.26493/1855-3974.1332.b49.

[6] L. Morgan, J. Morris and G. Verret, A finite simple group is CCA if and only if it has no element of order four, J. Algebra $\mathbf{5 6 9}$ (2021), 318-333, doi:10.1016/j.jalgebra.2020.10.028.

[7] J. Morris, Automorphisms of circulants that respect partitions, Contrib. Discrete Math. 11 (2016), 1-6, doi:10.11575/cdm.v11i1.62390.

[8] S. Project, Sagemath mathematics software system, 2017, http://www. sagemath.org.

[9] The GAP Group, Gap - groups, algorithms and programming, 2017, https://www . gap-system.org.

[10] A. T. White, Graphs of groups on surfaces, volume 188 of North-Holland Mathematics Studies, North-Holland Publishing Co., Amsterdam, 2001, interactions and models, https://www.elsevier.com/books/graphs-of-groups-on-surfaces/ white/978-0-444-50075-5. 